\title{
Conoscere il passato per guardare al futuro
}

\author{
Yuri Battaglia
}

\author{
U.O.C. di Nefrologia, Azienza Ospedaliero-Universitaria di Ferrara, Cona (Fe)
}

\begin{abstract}
KNOW THE PAST FOR LOOKING TO THE FUTURE

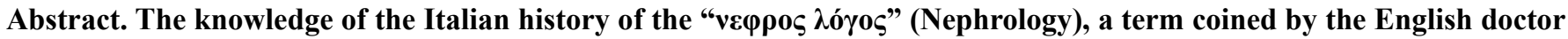
A.A. Osman (1883-1972), is of fundamental importance for a young doctor approaching this fascinating specialty. He/ she could thus appreciate the enthusiasm and the efforts made throughout history by the founders of this specialty for developing a new field of medicine that was largely unknown by then. In this article we will go through the milestones of Nephrology: form its birth, with the first rudimentary anatomical and pathophysiological studies and the development of the first dialysis techniques, up to the first kidney transplant performed in Italy. Over the centuries, many Italian researchers have given a decisive contribution to this fascinating story, gaining knowledge for the care and management of patients with renal diseases.
\end{abstract}

Key words: History, Italian nephrology, State-of-the-art

Conflict of interest: None.

Ricevuto: 9 Luglio 2013; Accettato: 25 Luglio 2013

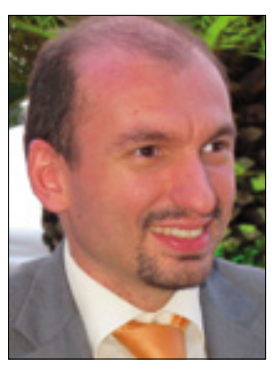

Yuri Battaglia
I primi studi di rilievo in campo nefrologico furono effettuati da Paracelso (1493-1541) nel 1527, in merito alla presenza di proteine nelle urine in pazienti edematosi e, successivamente, dall'italiano Cotugno (1736-1882) che, in quel di Napoli, descrisse la proteinuria dei pazienti con sindrome nefrosica e coniò il termine "albuminuria", poiché l'urina riscaldata mostrava un precipitato bianco e denso come l'albume dell'uovo (1). I primi studi di fisiologia renale, invece, risalgono al XXVII secolo e furono effettuati da due italiani, Malpighi (1628-1694) e Bellini (1643-1704), che, con microscopi rudimentali, scoprirono rispettivamente il glomerulo e i dotti $(2,3)$. Solo due secoli dopo, l'oculista Bowman (1816-1892) descrisse in modo dettagliato la struttura del glomerulo con all'interno i capillari e la sua connessione con i tubuli, mentre Ludwin (1816-1895) ipotizzò i processi di filtrazione glomerulare e di riassorbimento tubulare. Nella metà degli anni ' 90 , gli studi condotti dall'italiano Andreucci nei laboratori americani di Dallas di D.W. Seldin e F.C. Rector dimostrarono la presenza dell'equilibrio di filtrazione glomerulare mediante tecniche di micropuntura renale (4).

Gli studi sull'urea urinaria correlata all'introito di carne furono avviati dall'inglese L.S. Bale già nella metà dell'Ottocento; egli suggerì l'utilizzo di una dieta ipoproteica per ridurre i sintomi correlati all'uremia. Questi dati furono confermati successivamente anche da D. Volhard (1872-1950) agli inizi del Novecento ma, solo nel 1963, fu studiata a fondo la dieta ipoproteica dagli italiani C. Giordano, S. Giovanetti e Q. Maggiore.

Quest'ultimo suggerì al Cavalier Buitoni di brevettare la pasta aproteica ma, a causa degli alti costi di produzione, il brevetto fu venduto alla Dieterba, una delle più importanti case produttrici di alimenti aproteici del mercato italiano.

Il primo trattamento emodialitico fu effettuato nel 1947 da Fieschi (1904-1961), con un rene artificiale identico a quello di Kolff, ma ebbe uno scarso successo, date le gravi complicanze cardiovascolari (5).

Una decina di anni dopo, Battezzati (1912-1992) e Taddei (1923-1987) realizzarono un nuovo macchinario che diede ottimi risultati principalmente per il trattamento dei pazienti con insufficienza renale acuta. Il rene consisteva in 3 grossi cilindri, attorno ai quali si avvolgeva un grosso tubo di cellophane, immersi in una vasca con 210 litri di liquido di dialisi, preparato al momento; i sali venivano pesati con una comune bilancia e versati nella grossa vasca, che era riempita con acqua corrente da un comune rubinetto; con le mani nude si mescolava la soluzione per sciogliere i sali, contando sull'impermeabilità della membrana dializzante a batteri e tossine! Il sangue circolava in virtù di due pompe che lo aspiravano e reinfondevano nella vena cava inferiore mediante un catetere a due vie inserito nella vena femorale. Occorrevano 4 ore per preparare il rene e $1200 \mathrm{~mL}$ di sangue per riempire i tubi di cellophane (1). 
Nel 1971, Cambi a Parma istituì, invece, la dialisi breve, quattro ore al giorno per tre volte alla settimana, per i pazienti cronici con insufficienza renale cronica avanzata.

Nel 1966, il chirurgo italiano P. Stefanini a Roma effettuava il primo trapianto italiano renale e il terzo tra quelli effettuati in Europa. Un rene prelevato da una signora abruzzese venne impiantato in una ragazza romana di diciassette anni.

La prima società scientifica nefrologica la "Renal Association" fu fondata da A.A. Osman nel 1950 e, dopo circa sette anni, il Prof. Migone a Parma ideò e realizzò la Società Italiana di Nefrologia (SIN) insieme al Prof. Monasterio.

Nel 2001, nasceva la Fondazione Italiana del Rene (FIR) a Napoli grazie al Prof. Andreucci, con l'intento di riunire tutti coloro che erano coinvolti nelle problematiche di origine renale: medici, infermieri, pazienti, tecnici e così via, per prevenire le malattie renali, migliorare la qualità di vita dei pazienti e raccogliere fondi per la ricerca italiana.

J. Kople, presidente della International Federation of Kidney Foundation (IFKF), ideò, con il supporto della International Society of Nephrology (ISN), la Giornata Mondiale del Rene nel 2005 e diede mandato alla FIR insieme alla SIN di curare per l'Italia questo ambizioso progetto di prevenzione delle malattie renali mediante misurazioni della pressione arteriosa e controllo delle urine.

Alla luce delle gravi condizioni economiche attuali del nostro Paese, dei costi notevoli della Sanità e del tentativo di riassorbimento nella Medicina Interna, la lettura delle tappe fondamentali della nefrologia italiana può sicuramente riaccendere nel giovane nefrologo quell'amore profondo e quella passione sincera che ha dentro di sé, necessari per studiare, aggiornarsi ed effettuare un periodo all'estero per acquisire una corretta mentalità di ricerca scientifica e lavorare sempre di più per contribuire in modo reale all'ulteriore sviluppo di questa disciplina, così come hanno fatto in modo eccelso i padri fondatori.

\section{Riassunto}

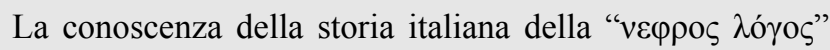
(Nefrologia), termine coniato dal medico inglese A.A. Osman (1883-1972), è di fondamentale importanza per un giovane che si avvicina a questa affascinante specialità. Attraverso la storia, infatti, si comprendono gli entusiasmi e gli sforzi fatti dai fondatori per far sviluppare un nuovo campo della medicina fino ad allora ben poco conosciuto. In questo articolo si ripercorrono le tappe fondamentali della nascita della nefrologia, dai primi rudimentali studi anatomici e fisiopatologici allo sviluppo delle prime tecniche dialitiche, fino al primo trapianto renale effettuato in Italia. È una storia affascinante, in cui molti ricercatori italiani, nel corso dei secoli, hanno dato un loro determinante contributo all'acquisizione delle conoscenze necessarie per la cura e la gestione dei pazienti nefropatici.

Parole chiave: Storia, Nefrologia italiana, Attualità

Dichiarazione di conflitto di interessi: L'Autore dichiara di non avere conflitto di interesse.

\author{
Indirizzo degli Autori: \\ Dr. Yuri Battaglia \\ U.O.C. di Nefrologia \\ Azienza Ospedaliero-Universitaria di Ferrara \\ Via A. Moro 8 \\ 44124 Cona (FE) \\ battagliayuri@gmail.com
}

\section{Bibliografia}

1. Andreucci V.E. 50 years of nephrology: between science and organizing activities. G Ital Nefrol. 2010 Jul-Aug;27(4):409-16.

2. Andreucci VE. About the glomerulus. Kidney Int 1972; 2: 349-51.

3. Bellini L. Opera Omnia. 2 Vol, 1708.

4. Andreucci VE, Herrera-Acosta J, Rector FC Jr, Seldin DW.
Effective glomerular filtration pressure and single nephron filtration rate during hydropenia, elevated ureteral pressure, and acute volume expansion with isotonic saline. J Clin Invest 1971; 50: 2230-4.

5. Fogazzi GB. Archivi storici della Nefrologia Italiana. 11 rene artificiale commissionato nel 1947 da Aminta Fieschi (19041991). G Ital Nefrol 2003; 20: 43-8. 